\title{
A 5-Year Review of Senecavirus A in China since Its Emergence in 2015
}

\author{
Fuxiao Liu ${ }^{\star}$, Qianqian Wang ${ }^{\dagger}$, Yilan Huang ${ }^{\dagger}$, Ning Wang and Hu Shan* \\ College of Veterinary Medicine, Qingdao Agricultural University, Qingdao, China
}

Senecavirus A (SVA), previously known as Seneca Valley virus, is classified into the genus Senecavirus in the family Picornaviridae. This virus can cause vesicular disease and epidemic transient neonatal losses in swine. Typical clinical signs include vesicular and/or ulcerative lesions on the snout, oral mucosa, coronary bands and hooves. SVA emerged in Guangdong Province of China in 2015, and thereafter gradually spread into other provinces, autonomous regions and municipalities (P.A.M.S). Nowadays more than half of the P.A.M.s have been affected by SVA, and asymptomatic infection has occurred in some areas. The phylogenetic analysis shows that China isolates are clustered into

\section{OPEN ACCESS}

Edited by:

Jesus Hernandez,

Consejo Nacional de Ciencia y

Tecnología (CONACYT), Mexico

Reviewed by:

Pablo Pineyro,

lowa State University, United States Santhamani Ramasamy,

Albert Einstein College of Medicine, United States

*Correspondence:

Fuxiao Liu

laudawn@126.com

Hu Shan

shanhu67@163.com

tThese authors have contributed equally to this work

Specialty section:

This article was submitted to

Veterinary Infectious Diseases,

a section of the journal

Frontiers in Veterinary Science

Received: 30 May 2020

Accepted: 21 August 2020

Published: 30 September 2020

Citation:

Liu F, Wang Q, Huang Y, Wang N and Shan H (2020) A 5-Year Review of Senecavirus $A$ in China since Its Emergence in 2015.

Front. Vet. Sci. 7:567792.

doi: 10.3389/fvets.2020.567792 five genetic branches, implying a fast evolutionary speed since SVA emergence in 2015. This review presented current knowledge concerning SVA infection in China, including its history, epidemiology, evolutionary characteristics, diagnostics and vaccines.

Keywords: senecavirus A, China, epidemiology, phylogenetic analysis, diagnostics, vaccines

\section{INTRODUCTION}

Senecavirus A (SVA), also known as Seneca Valley virus (SVV), belongs to the genus Senecavirus in the family Picornaviridae. SVA now is the only member in the genus Senecavirus, and has only one serotype (1). This virus was initially identified incidentally as a contaminant in cell culture medium during cultivation of PER.C6 (transformed fetal retinoblast) cells. SVA is not pathogenic to normal human cells, but has potent oncolytic activity in tumor cells, such as neuroendocrine tumor cells (2) and human retinoblastoma cells (3).

SVA can cause vesicular disease and epidemic transient neonatal losses (ETNL) in swine. In 2007, dozens of pigs at a Canada market showed idiopathic vesicular disease (IVD)-like clinical signs: broken vesicles along the coronary band that was swollen and blanched white, with tissues separating from the edge of hoof and dewclaws sloughing from their attachments. The IVD was subsequently diagnosed with SVA infection (4). In 2010, a 6-month-old intact male Chester White boar exhibited the IVD-like clinical signs in the USA, and then was diagnosed with SVA infection (5). At the end of 2014 and the beginning of 2015, frequent outbreaks of SVA infection were reported in weaned and adult pigs in different geographical regions of Brazil (6-9).

In 2015, vesicular lesions were observed in pigs at farms in Guangdong Province of China, where newborn piglets presented with sudden death at the same time. This case was diagnosed with SVA infection, the first outbreak in China (10). In the following years, SVA gradually spread into other provinces. By December 2019, more than half of the provinces, autonomous regions and municipalities (P.A.M.s) had been reported to be affected by SVA infection in China. The phylogenetic analysis shows that China isolates can be mainly grouped into five genetic branches (11). Events of genetic recombination have occurred among a few China strains (12-14), and asymptomatic SVA infection has also been found in some areas $(15,16)$. Here, we comprehensively reviewed a 5-year history (2015-2019) of SVA infection in China, including its history, epidemiology, evolutionary characteristics, diagnostics, and vaccines. 


\section{SVA CHARACTERISTICS}

\section{Viral Structure}

SVA is the only member in the genus Senecavirus, and shares many features with other picornaviruses. Morphologically, the mature virion of SVA is a non-enveloped icosahedral ( $\mathrm{T}=$ pseudo3) particle with a diameter of $\sim 27 \mathrm{~nm}$. Purified virion or, less probably, procapsid (17), reveals a spherelike shape under our observation by transmission electron microscopy (Figure 1a). The viral capsid is composed of a densely-packed icosahedral arrangement of 60 protomers, each consisting of four structural proteins (VP1, VP2, VP3, and VP4). The VP1, VP2, and VP3 are "spliced" together to form an outer shell (Figure 1b); and the VP4 is located beneath the internal side of the capsid (Figure 1c). A viral protein (VPg) is covalently linked to the $5^{\prime}$ end of viral RNA genome, encapsidated inside the virion (Figure 1c).

\section{Viral Genome}

The SVA genome is a positive-sense, single-stranded, nonsegmented, and linear RNA, 7.3 nt in length, with a $3^{\prime}$ poly (A) tail but without a $5^{\prime}$ capped structure (Figure 1d). The genome contains $5^{\prime}$ and $3^{\prime}$ untranslated regions (UTR), and a single long open reading frame (ORF) of polyprotein precursor. The $5^{\prime}$ UTR contains a sequence of type IV internal ribosome entry site (IRES) (18), which has structural and functional similarities to those of pestiviruses (Figure 1d) (19), allowing for translation initiation in a cap-independent manner. The $3^{\prime}$ UTR is $\sim 70 \mathrm{nt}$ in length, and reveals a kissing-loop structure, followed by a poly (A) sequence. The polyprotein ORF consists of the leader (L) and

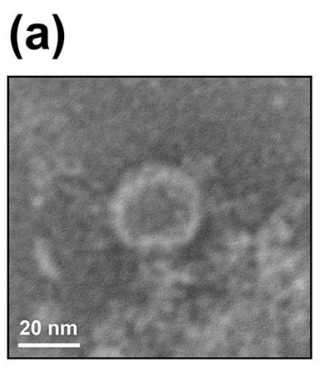

(b)

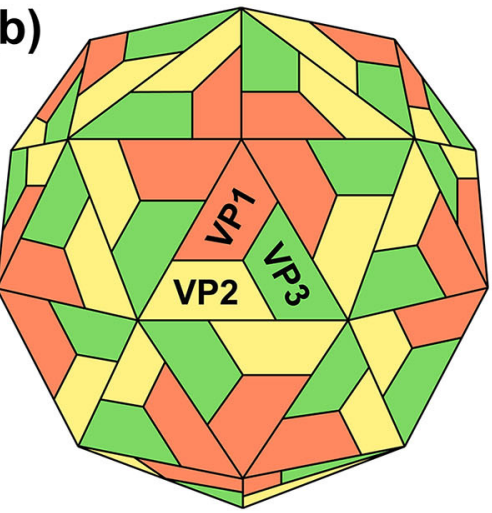

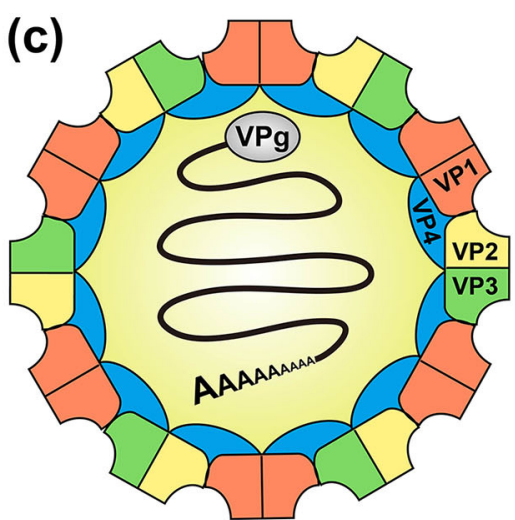

(d) IRES

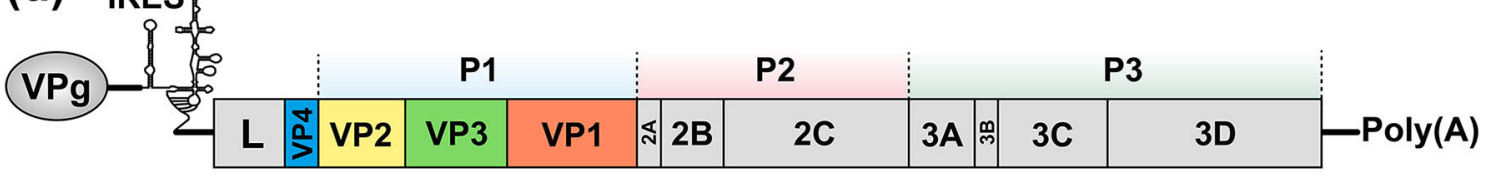

(e)
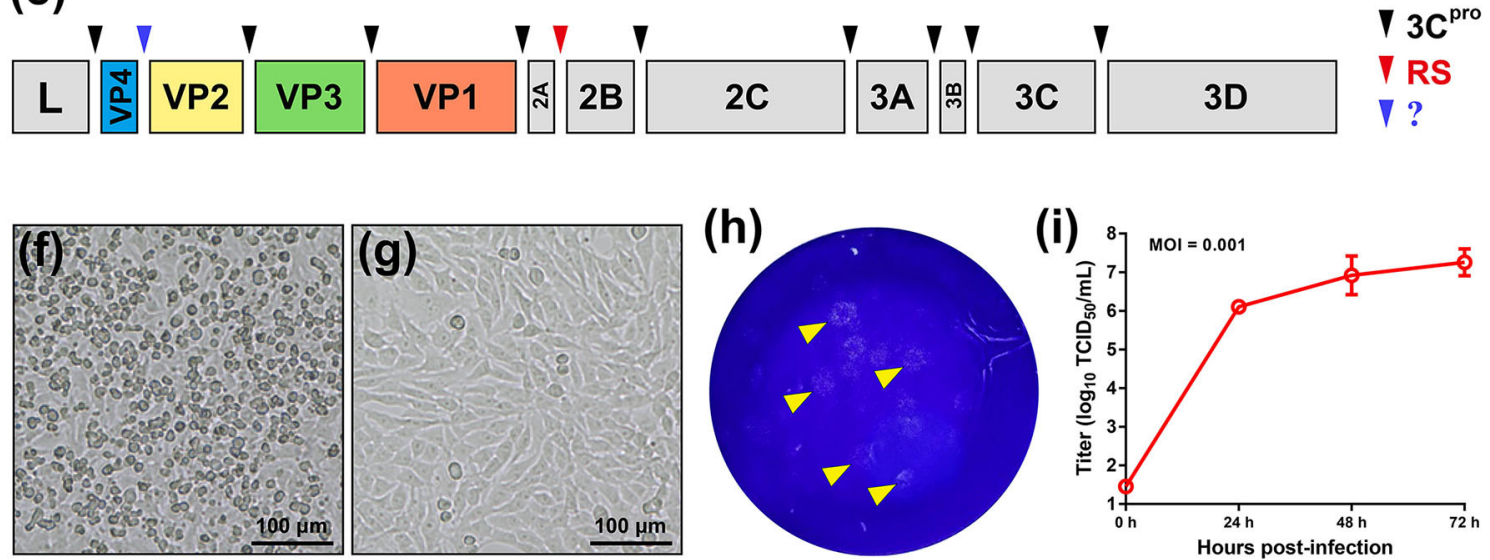

FIGURE 1 | Characteristics of Senecavirus A. Transmission electron microscopy of purified SVA virion (a). Schematic representations of SVA virion: front (b) and section (c) views. Schematic representation of SVA genome (d). Cleavage sites (arrow-headed) within SVA polyprotein (e). Typical cytopathic effect, cell rounding, at $24 \mathrm{~h}$ post infection with SVA (f), and non-infected cell monolayer as mock (g). Plaque formation (arrow-headed) of SVA-infected cell monolayer (h). Multi-step growth curve (MOI $=0.001$ ) of rescued SVA CH-LX-01-2016 cultivated in BSR-T7/5 cells (i). IRES, internal ribosome entry site; 3C ${ }^{\text {pro }}$, 3C protease; RS, ribosomal skipping; ?, unknown. 
3 major protein regions (P1, P2, and P3), and follows the standard "L-4-3-4" layout, namely "Leader -4 polypeptides of $\mathrm{P} 1-3$ polypeptides of $\mathrm{P} 2-4$ polypeptides of $\mathrm{P} 3$ " or "L-VP4-VP2-VP3VP1-2A-2B-2C-3A-3B-3C-3D". The first SVA isolate from China (20), CH-01-2015 (Genbank access No.: KT321458), has an $~ 7,300$-nt-long genome, which contains a 668 nt $5^{\prime}$ UTR, a 64 nt $3^{\prime}$ UTR and a 6,543 nt ORF that encodes a 2,181 aa polyprotein.

\section{Viral Proteins}

Referring to other picornaviruses (21), a polyprotein precursor of SVA can be stepwisely cleaved into twelve polypeptides (Figure 1e). The primary "cleavage" event is involved in a mechanism of ribosomal skipping, and occurs at the conserved TNPG $\downarrow \mathrm{P}$ motif between the $2 \mathrm{~A}$ and the $2 \mathrm{~B}$, in which the arrow indicates the "cleavage" site. The $2 \mathrm{~A}$ performs the function of ribosomal skipping. In the $\mathrm{P} 1$ region, the $\mathrm{P} 1$ polypeptide is cleaved into VP0, VP3, and VP1 by the 3C protease. The precursor VP0 will be cleaved further into VP4 and VP2, and however the cleavage mechanism remains unclear. The VP1, VP2, VP3, and VP4 are structural proteins, required for encapsidation of SVA genome. The $\mathrm{P} 2-\mathrm{P} 3$ region is cleaved into seven non-structural proteins: 2A, 2B, 2C, 3A, 3B (VPg), $3 \mathrm{C}$ (protease), and $3 \mathrm{D}$ (polymerase). The $3 \mathrm{~B}$ is the genomelinked polypeptide, sharing a low homology with those of other picornaviruses, and functions as a protein primer for RNA synthesis. The $3 \mathrm{C}$ is a protease, responsible for cleaving the SVA polyprotein precursor. The $3 \mathrm{D}$ is a RNA-dependent RNA polymerase, functioning in virus replication and VPg uridylylation (22).

\section{Growth Characteristics in vitro}

Many cell lines, such as BHK-21 (baby hamster kidney-21), PK-15 (porcine kidney-15) and ST (swine testis), can be used for isolation and cultivation of SVA (23). SVA-inoculated cells would exhibit typical cytopathic effects (CPE), like cell rounding and detachment (Figure 1f), compared with a non-infected cell monolayer (Figure 1g). Plaque morphologies generally depend on SVA isolates and cell lines. Figure $\mathbf{l h}$ shows SVA-induced plaque formation on a BSR-T7/5 cell monolayer at $72 \mathrm{~h}$ post infection (hpi). SVA replication is able to reach a high level of peak titer in its susceptible cells. Wang et al. (24) recently compared growth kinetics of five SVA isolates from Guangdong Province of China. All of them displayed similar growth kinetics to one another in the ST-R cell line, and could reach their peak titers $\left(10^{8}-10^{9} \mathrm{TCID}_{50} / \mathrm{mL}\right)$ at $48 \mathrm{hpi}(11)$. We recently rescued a China strain (CH-LX-01-2016) using reverse genetics, and its growth kinetics was measured during 0 and 72 hpi in BSR-T7/5 cells (Figure 1i). It subsequently underwent serial 80 passages in vitro for genetic analysis by next-generation sequencing, which revealed neither sequence-deleting nor -inserting phenotype detectable in viral progenies, suggesting a relatively high stability of viral nucleic acids during consecutive passages.

\section{Viral Pathogenesis}

Clinical signs of SVA infection are indistinguishable from those of other vesicular diseases, caused by foot-and-mouth disease virus (FMDV), swine vesicular disease virus, vesicular stomatitis

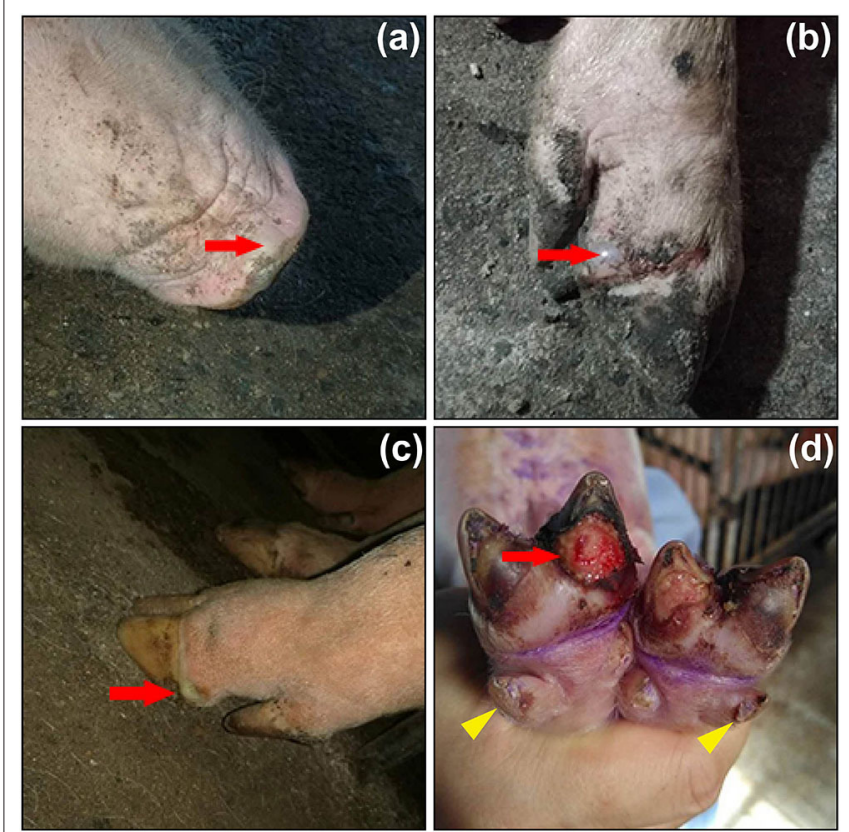

FIGURE 2 | Clinical signs of pigs infected with Senecavirus A in Fujian Province of China in 2017. Fluid-filled vesicles on snout (a, red arrow) and coronary bands (b,c, red arrows). Ruptured vesicle with ulceration and erosion beneath the toe (d, red arrow), and lesions on dewclaws (d, yellow arrowheads). Adapted from the reference (23) with permissions of the Elsevier (License No.: 4892830654384).

virus and vesicular exanthema of swine virus (1). Typical clinical signs are vesicular and/or ulcerative lesions on the snout, oral mucosa, coronary bands and hooves (Figure 2). Rupture of a fluid-filled vesicle would leave erosion on the skin, subsequently evolving to form a crust on the affected area. Some herds additionally suffer an increase in neonatal losses of 1- to 4day-old piglets, termed ETNL (25). Other clinical signs include lethargy, lameness, anorexia and even diarrhea.

Experimental infection of SVA in finishing pigs revealed that tonsil was one of the primary sites of SVA replication during the acute stage of infection. The viremia period was relatively short, lasting $\sim 7$ days, whereas virus shedding was detected between 1 and 28 days post infection in oral and nasal secretions and feces. Some historical strains, albeit detected in pigs presenting with vesicular disease, failed to reproduce the disease in virus-inoculated pigs (26).

\section{EMERGENCE, SPREAD, AND INFLUENCE OF SVA IN CHINA}

\section{First Outbreak in 2015}

In March 2015, an outbreak of vesicular disease occurred in Guangdong Province of China. The clinical signs were mainly characterized by vesicular lesions in sows and acute death of neonatal piglets. Conventional differential diagnoses were carried out to exclude foot-and-mouth disease, swine vesicular disease, vesicular exanthema of swine and vesicular stomatitis. Wu et al. 
(10) designed seven pairs of primers for amplifying the complete genome of SVA by RT-PCR assays. Sequence alignment showed that the emerging virus shared 94.4-97.1\% nucleotide identities with eight SVA strains (10). This was the initial report of SVA infection in China. The first China isolate was the CH-01-2015 (20), clustered in the same clade as that of the USA strains. The earliest isolates from Guangdong Province, including the $\mathrm{CH}-01$ 2015, CH-02-2015, CH-04-2015, CH-DB-11-2015, CH-LX-012016, CH-DL-01-2016, and CH-ZW-01-2016, were clustered into two different clades by a phylogenetic analysis. At the nucleotide level, they shared 96.3-97.1 and 97.9-98.3\% similarities with the USA and Brazil strains, respectively (27).

\section{SVA Spread since 2015}

Since its emergence in Guangdong, the SVA continuously spread into other P.A.M.s. The second SVA-affected province was Hubei in March 2016, when an outbreak of vesicular disease occurred in piglets at a pig farm (28). An infectious SVA was isolated and named HB-CH-2016 (Genbank access No.: KX377924), which shared an extremely high (99\%) homology of genome with the CH-01-2015, implying a significant epidemiological correlation between the first two events of SVA outbreak in China. In December 2016, finishing pigs exhibited typical signs of vesicular disease at a farm of Heilongjiang Province in northeast China. A SVA strain was isolated and designated SVA/HLJ/CHA/2016 (Genbank access No.: KY419132) (29). Its genome shared high nucleotide identities (93.8-99\%) with others deposited in Genbank. Interestingly, it was genetically closer to the 2015 USA strains than to those reported earlier in China.

In January 2017, SVA emergence was almost simultaneously found in two non-neighboring provinces, Fujian and Henan (30). Three strains, CH-FJ-2017 (Genbank access No.: KY747510), CH-HN-2017 (Genbank access No.: KY747511) and CH-HNSL2017 (Genbank access No.: KY747512) were subsequently isolated for phylogenetic analysis. The nucleotide sequence identities ranged from 99.7-99.8\% among the three isolates. They shared the highest homologies of genome (98.8-98.9\%) and polyprotein (99.5-99.6\%) with the KS15-01 strain, rather than with the earlier China isolates. Meanwhile, other two strains, $\mathrm{CH} / \mathrm{FuJ} / 2017$ (23) and CH-HNKZ-2017 (31), were isolated from Fujian and Henan Provinces, respectively. Both isolates were proven to share the highest genetic homologies with two USA isolates, USA/GBI29/2015 and US-15-40381IA, respectively. In the same year, SVA infection was identified at a pig farm in Gansu Province (32), which was not adjacent to other provinces affected by SVA earlier. A phylogenetic analysis showed that the Gansu isolate CH-LZ-2017 shared the highest nucleotide homology (99\%) of VP1 with that of the Henan strain CH-HN-2017.

In August 2018, SVA emerged in Guangxi, a neighboring province of Guangdong. Phylogenetic analyses indicated that the Guangxi isolate CH-GX-01-2018 shared the highest homology (98.6\%) with those isolated from Guangdong in 2017 at the genome level (33), and the other Guangxi isolate CH-GX-022018 also revealed the highest genetic identity with several Guangdong strains (34), implying a possible epidemiological relationship between Guangxi and Guangdong.

\section{SVA Reemergence}

It was recently reported that SVA reemerged in some provinces, especially in Guangdong. In 2017, four groups independently isolated and further analyzed reemerging strains in Guangdong. The first group isolated 17 novel strains, and revealed their complete genomes, VP1, 3C, and 3D genes sharing 96.5-99.8, 95.1-99.9, 95.6-100, and 96.9-99.7\% nucleotide identities with one another, respectively (35). The second group isolated six strains, and revealed nucleotide identities among their genomes ranging from 99.6 to $99.8 \%$. These six strains shared the highest genomic identity with the USA/GBI29/2015 (98.5-98.6\%), and were notably distinct from earlier isolates from China (36).

The third group determined two strains, CH-GD-2017-1 and CH-GD-2017-2, both of which showed different characteristics from those of earlier isolates in Guangdong, but were more similar to the USA strains (37). The forth group isolated five strains, GD01/2017, GD03/2017, GD04/2017, GD05/2017, and GD06/2017, subsequently subjected to genomic sequencing. The result revealed that GD01/2017 and GD03/2017 shared an extremely high homology with each other (99.4\%), were both closely related to the USA/GBI29/2015 (98.6\%), but showed relatively low homologies with GD04/2017, GD05/2017, and GD06/2017. All five isolates were classified into four different genetic clades by a phylogenetic analysis, suggesting a low genetic homology among them, except that between GD01/2017 and GD03/2017. Such a great diversity among their genomes implied that local strains in Guangdong had evolved into different branches (11).

\section{Current Status of SVA Infection}

In addition to Guangdong, Hubei, Heilongjiang, Fujian and Henan, other P.A.M.s have actually been affected by SVA. During 2016 and 2018, the China Animal Health and Epidemiology Center (CAHEC) collected hundreds of samples from 15 P.A.M.s, including Hunan, Yunnan, Xinjiang, Liaoning, Fujian, Hubei, Guangxi, Hebei, Jiangxi, Jilin, Sichuan, Shandong, Shannxi, Shanghai, and Guizhou. More recently, the CAHEC reported the result of detection for all samples, showing that, out of these 15 P.A.M.s, only four provinces, Hebei, Jiangxi, Jilin, and Shannxi, were not affected by SVA infection (38). As to Jilin, a recent report, nonetheless, revealed that pigs actually had been subclinically infected by SVA at a few farms in this province (39).

Unlike other P.A.M.s, Hainan Province is an ocean island, and therefore not prone to be affected by animal diseases. A report, nevertheless, revealed that out of 2,547 serum samples collected from Hainan in 2018, 278 were diagnosed SVA antibody-positive by ELISA analysis (40). Based on existing reports, we created a map that exhibited geographical distribution of SVA-affected P.A.M.s in China during 2015 and 2019 (Figure 3), and key data were listed in Table $\mathbf{1 .}$

\section{Phylogenetic Analysis of SVAs}

Phylogenetic analysis of global SVA genomes revealed two major evolutionary clusters, USA- and Canada-like strain clusters. To date, more than half of the P.A.M.s have been affected by SVA in China (Figure 3). Almost all isolates from these P.A.M.s are classified into both clusters. Additionally, all China isolates could 


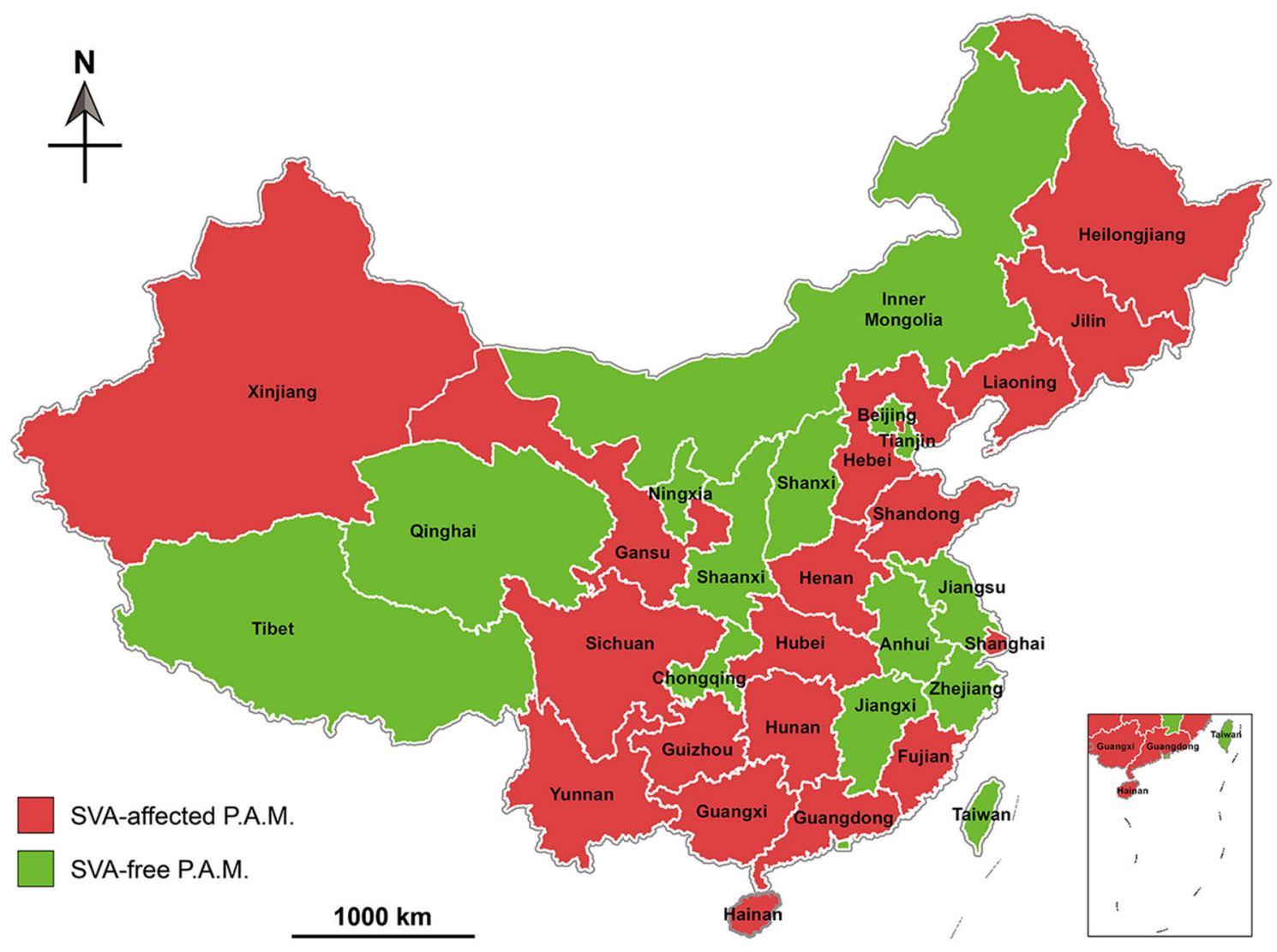

FIGURE 3 | Geographical distribution of Senecavirus A-affected and -free P.A.M.s in China during 2015 and 2019. P.A.M, province, autonomous region and municipality.

be mainly grouped into five genetic branches, namely clade I, II, III, IV, and V (11). Here, we constructed a phylogenetic tree (Figure 4), by which a part of China isolates were compared with exotic ones for showing their evolutionary relationship. The phylogenetic analysis reveals that clade I, II, III, and IV belong to the USA-like cluster, and only the clade V belongs to the Canadalike cluster, suggesting that the USA-like strains are predominant in China. A total of five clades in Figure 4 demonstrate that the current genetic relationship become more complex than the past one among China isolates. SVA has been evolving in China since its emergence in 2015.

\section{Recombination Events Between SVAs}

SVA is one of RNA viruses, characterized by relatively high rate of genetic mutation during their replication in vitro and in vivo (42). Genomic recombination by a copy-choice mechanism is a genetic feature of picornaviruses (43), and unfortunately is a widely neglected issue during evolutionary analysis of variants. In addition, picornaviruses generally have a poor ability to accommodate stably foreign genes. Using reverse genetics, we previously rescued two recombinant SVAs, both of which separately expressed a green fluorescent protein (44) and a luciferase. It has been demonstrated that both recombinant
SVAs are genetically unstable and prone to losing their foreign sequences during serial passages in vitro.

SVA has been continually evolving, and even recombining with one another in field since its emergence in China. A SimPlot analysis revealed two breakpoints that separated the genome of China SVA isolate $\mathrm{HeN}-1 / 2018$ into three regions, out of which two (1-959 and 2,355-7,312 nt) derived from the USA/IA44952/2015, and the other (960-2,354 nt) derived from the USA/IN_Purdue_4885/2015 (14). The SimPlot analysis implied a possible even of recombination between both USA strains (or their progenies) to form the emerging China strain. Liu et al. (12) isolated two novel SVAs, CH-GDFS-2018 and CHGDJY-2018, from slaughtered pigs, which had recovered from the vesicular disease. Their recombinant fragments comprised both structural and non-structural protein regions, involved in genetic recombination between local (or domestic) and foreign strains (12).

More recently, Guo et al. (13) reported that one isolate (HeNNY-1/2018) and two previously reported strains (HB-CH2016 and SVA/CHN/10/2017) were identified as recombinants using several algorithms. It revealed that the recombination among SVA strains had occurred in China since 2016 or earlier (13). The frequent recombination events caused emergence of novel variants, consequently increasing the complexity of SVA 
TABLE 1 | SVA-affected provinces, autonomous regions and municipality in China during 2015 and 2019.

\begin{tabular}{lcllr}
\hline P.A.M. & $\begin{array}{c}\text { Year of } \\
\text { SVA emergence }\end{array}$ & $\begin{array}{l}\text { Representative } \\
\text { isolate }\end{array}$ & $\begin{array}{l}\text { Genbank } \\
\text { access No. }\end{array}$ & References \\
\hline Guangdong & 2015 & CH-01-2015 & KT321458 & $(20)$ \\
Hubei & 2016 & HB-CH-2016 & KX377924 & $(28)$ \\
Heilongjiang & 2016 & SVA/HLJ/CHA/2016 KY419132 & $(29)$ \\
Fujian & 2017 & CH-FJ-2017 & KY747510 & $(30)$ \\
Henan & 2017 & CH-HN-2017 & KY747511 & $(30)$ \\
Gansu & 2017 & CH-LZ-2017 & NA & $(32)$ \\
Guangxi & 2018 & SVA/GX/CH/2018 & MK039162 & $(33)$ \\
Shandong & NA & SW-CH-SD & MH779611 & $(41)$ \\
Hainan & 2018 & NA & NA & $(40)$ \\
Xinjiang & NA & NA & NA & $(38)$ \\
Liaoning & NA & NA & NA & $(38)$ \\
Hunan & NA & NA & NA & $(38)$ \\
Yunnan & NA & NA & NA & $(38)$ \\
Sichuan & NA & NA & NA & $(38)$ \\
Shanghai & NA & NA & NA & $(38)$ \\
Guizhou & NA & NA & NA & $(38)$ \\
Jilin & NA & NA & NA & $(39)$ \\
\hline PAM & & NA &
\end{tabular}

P.A.M., province, autonomous region and municipality; NA, not available.

spread in China. Considering the adaptation of SVA to pigs, the occurrence of natural recombination is of clinical importance.

\section{Pathogenesis of China Isolates}

Four groups independently reported experimental infection of SVA China isolates in animals. The first group inoculated pigs with two SVA isolates, GD-S5/2018 and GD04/2017. The result showed that the GD-S5/2018 was a virulent isolate, while the GD04/2017 was nearly avirulent. Both strains shared $93.9 \%$ nucleotide identity with each other, whereas the mechanism, involved in impact of genomic variance on virulence, remained unclear (45). The second group compared other two isolates, HB$\mathrm{CH}-2016$ and $\mathrm{CH} / \mathrm{AH}-02 / 2017$, for evaluating their pathogenesis in pigs. The result revealed that SVA-infection-like clinical signs were observed only in CH/AH-02/2017-infected pigs (46). Both isolates shared $96 \%$ homology with each other at the genomic level, and exhibited 29 amino acid differences. The HB-CH2016 and $\mathrm{CH} / \mathrm{AH}-02 / 2017$ belonged to the Canada- and USAlike strains, respectively. The USA-like strains have become predominant among China isolates since 2017.

The third group inoculated 30-35-, 55-65-, and 90-100-dayold pigs with a clinical isolate (SVV-CH-SD) from Shandong Province. The result revealed that 90-100-day-old pigs developed low-grade fever, blisters and lameness, whereas neither 30-35nor 55-65-day-old pigs showed typical clinical signs, suggesting the SVV-CH-SD with age-dependent variation in virulence (41). The forth group isolated one SVA (SVA-FJLY strain) from Fujian Province in 2018, with which subsequently three pigs were intranasally inoculated $\left(10^{9} \mathrm{TCID}_{50} / \mathrm{pig}\right)$. Two SVA-infected pigs developed vesicular and ulcerative lesions on their snouts and oral mucosa, and the other had no typical clinical signs (47).
The results from these four groups suggested that high- and lowvirulence SVAs were simultaneously circulating in China. Indeed, subclinically infected cases are growingly identified in China.

\section{DIAGNOSTICS AND VACCINES DEVELOPED IN CHINA}

\section{Diagnostics}

A variety of conventional and novel methods have been established for diagnosing SVA infection. Non-Chinese groups have developed two kinds of major methods, competitive ELISA $(48,49)$ and distinct RT-PCRs $(50-56)$. Some of them have been used as commercially available kits for clinical detection.

Meanwhile, Chinese researchers also reported nucleic acidbased methods, characterized by high specificity and sensitivity, and having a potential in clinical detection of SVA. These methods mainly include conventional RT-PCR (57), quantitative RT-PCR (qRT-PCR) (24, 58), RT-droplet digital PCR (RTddPCR) (59), reverse transcription loop-mediated isothermal amplification (RT-LAMP) (60), RT-LAMP-lateral flow dipstick (RT-LAMP-LFD) (61), recombinase polymerase amplification (RPA) and RPA-LFD (62). Their detailed data are listed in Table 2.

Of all the above-mentioned methods, the qRT-PCR is now most broadly used as a nucleotide-based method for clinical detection. The novel RT-ddPCR is a third-generation PCR technique, and its detection limit was $1.53 \pm 0.22$ copies of SVA RNA/reaction, $\sim 10$-fold greater sensitivity than that of the qRT-PCR (59). RPA is another novel tool, a highly sensitive and selective isothermal amplification technique for detecting viral nucleic acids. The lowest concentration of 28 copies of SVA $\mathrm{RNA} / \mu \mathrm{L}$ could be detected within $10 \mathrm{~min}$ at $40^{\circ} \mathrm{C}$ (63). These methods have their own relative merits, can complement but cannot substitute for one another.

Besides the nucleotide-based methods, serological diagnostics also plays a key role in clinical detection of SVA. The joint use of both methods would enhance the accuracy of diagnosis. Zhao et al. (64) developed a VP1-based indirect ELISA, which could be used for detection of SVA-induced antisera, and conferred no cross-reaction with those of common swine viruses (64). In addition to the VP1, the VP2 could also be used for establishment of serum diagnostics. The VP2-based block and indirect ELISAs showed high sensitivity and specificity for anti-SVA sera $(65,66)$. Using the recombinant SVA that expressed a green fluorescent protein, we recently developed a rapid virus neutralization test (VNT) for detecting serum samples. The novel VNT shortened the period of 96-well plate incubation to $48 \mathrm{~h}$, much less than that of the conventional VNT. Moreover, it was more sensitive and specific than the conventional VNT using wide-type SVA, because a final VNT result of readout would depend on green fluorescence, rather than CPE (44).

\section{Vaccines}

Compared with the numerous studies of diagnostics, those of SVA vaccines have been rarely reported as yet. Despite no commercially available vaccines now, the unique SVA serotype makes the applicable scope of a given candidate broader than 


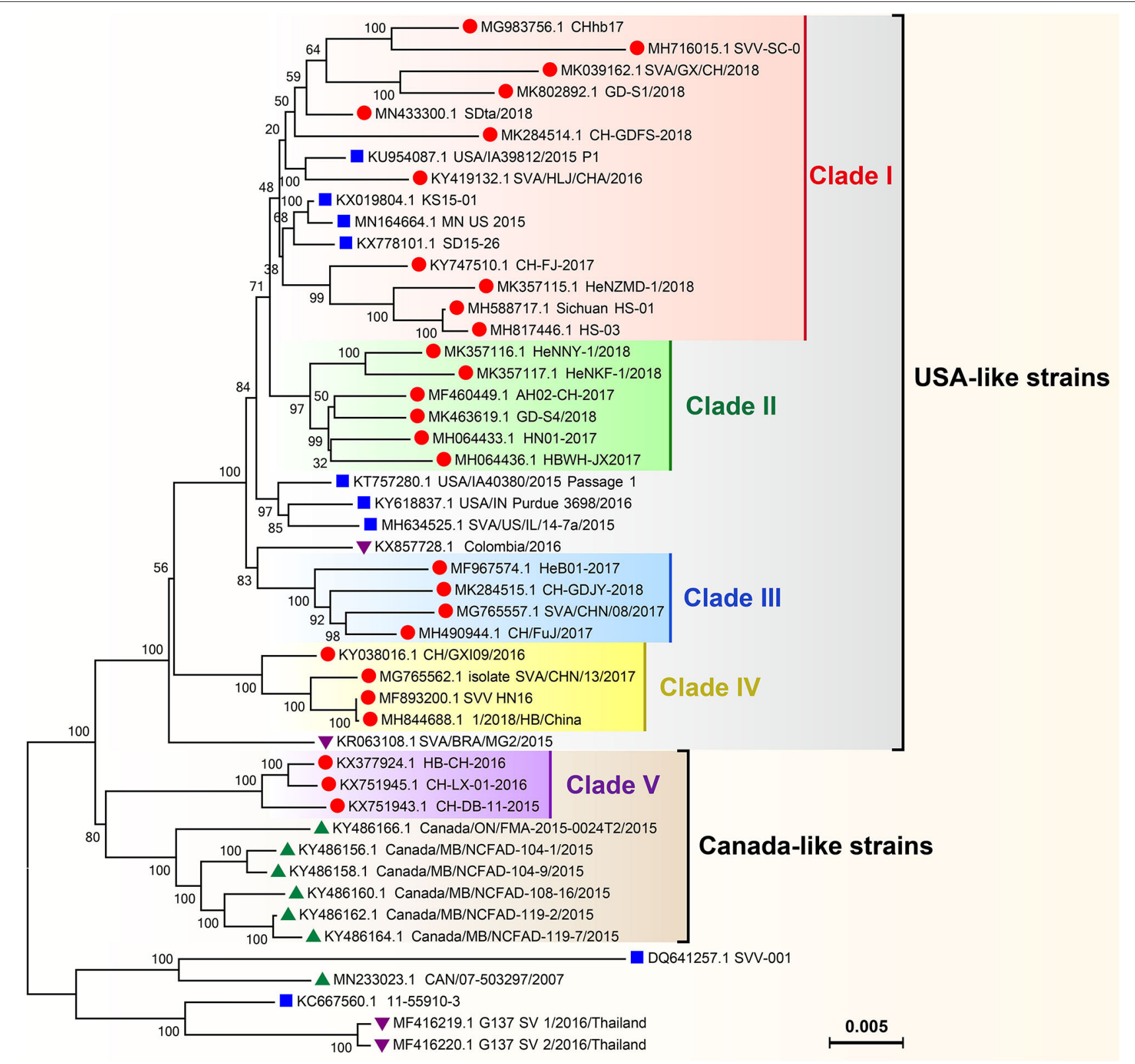

FIGURE 4 | Phylogenetic analysis on genomes of SVA isolates. The Neighbor-joining method and Kimura-2-parameter model were used for construction of the phylogenetic tree using MEGA 7.0 software. Numbers indicated the bootstrap value (1,000 replicates). SVA isolates from China, the USA, Canada and other countries

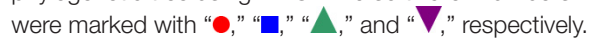

those of multi-serotype viruses. Moreover, the SVA can efficiently propagate in BHK-21 cells to reach a high titer, even more than $10^{9} \mathrm{TCID}_{50} / \mathrm{mL}$ in culture supernatant, greatly facilitating mass production of seed stocks. Sharma et al. (67) developed a novel candidate of live-attenuated vaccine, with which a single immunization could confer protection against heterologous SVA challenge, as demonstrated by absence of overt disease and reduced viremia, virus shedding and viral load in tissues (67). This candidate represents a promising alternative to prevent SVA infection in pigs.
Besides the live-attenuated candidate, inactivated and DNAbased vaccines also showed the potential in stimulating immune responses. A Chinese group developed an inactivated vaccine candidate using the isolate CH-FJ-2017. The seed virus was produced in BHK-21 cells by roller bottles, inactivated with binary ethylenimine, and emulsified in oil adjuvant. The immunogenicity of inactivated SVA was evaluated in pigs by VNT. The vaccinated pigs ( $2 \mu \mathrm{g} /$ dose) could develop hightiter neutralizing antibodies, and showed no clinical signs when challenged with the homologous virus, indicating a 
TABLE 2 | Nucleic acid-based diagnostics of SVA infection developed in China.

\begin{tabular}{|c|c|c|c|c|}
\hline Diagnostics & Target sequence & Limit of detection & No cross-reaction with & References \\
\hline RT-PCR & $5^{\prime}$ UTR & $1 \mathrm{TCID}_{50} / \mathrm{mL}$ & EMCV, FMDV, PRV, PRRSV, CSFV, and PEDV & $(57)$ \\
\hline qRT-PCR & VP1 & 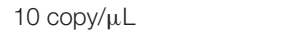 & VSV, APPV, PRV, and FMDV & $(58)$ \\
\hline qRT-PCR & 3D & 10.4 copy/ $\mu L$ & FMDV, PRRSV, PEDV, PCV2, and PRV & $(24)$ \\
\hline RPA & $3 \mathrm{D}$ & 28 copy/ $\mu \mathrm{L}$ & FMDV, CSFV, PRRSV, PEDV, and TGEV & $(63)$ \\
\hline RT-LAMP & VP1 and VP2 & $1 \mathrm{TCID}_{50} / \mathrm{mL}$ & PCV2, PEDV, CSFV, PRRSV, PRV, VSV, SVDV, and FMDV & $(60)$ \\
\hline RT-ddPCR & 3D & $1.53 \pm 0.22$ copy/reaction & FMDV, VSV, SVDV, PRRSV, CSFV, and PCV2 & $(59)$ \\
\hline RT-LAMP-LFD & 3D & 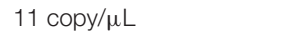 & FMDV, SVDV, VSV, PDCoV, PEDV, TGEV, PRRSV and CSFV & $(61)$ \\
\hline RPA-LFD & 3D & 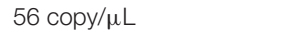 & FMDV, CSFV, PRRSV, PEDV, TGEV, PRV, and PCV & $(62)$ \\
\hline
\end{tabular}

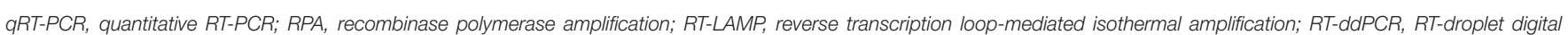

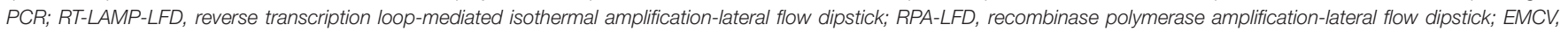

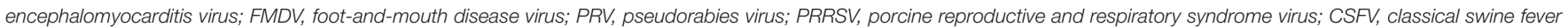

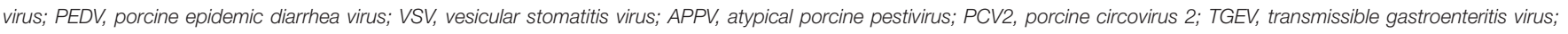
SVDV, swine vesicular disease virus; PDCoV, porcine deltacoronavirus.

high protective efficacy of the produced vaccine (68). More recently, this group constructed a recombinant plasmid, which not only efficiently expressed a fusion polypeptide, P12A$3 \mathrm{C}$, in $293 \mathrm{~T}$ cells, but also could induce high-titer SVAspecific antibodies in mice (69). FMDV virus-like particles (VLPs) were demonstrated to have the ability to induce robust immune responses in vivo (70-72), which would offer a new perspective on development of novel VLP vaccines against SVA (73).

Nowadays, there are two major challenges for the development of SVA vaccines. One is screening of standard strains with high virulence for challenge tests in pigs. Clinical signs cannot possibly be reproduced after experimental infection with some low-virulence SVAs, or even with those clinically isolated from diseased pigs. We found that a few strains, albeit isolated from vesicular fluid of diseased pigs, could not cause observable clinical signs, even obvious onset of pyrexia, in experimental pigs (data not shown). The other challenge is establishment of laboratory animal models susceptible to SVA infection. Laboratory animal models have provided valuable insight into the pathogenesis and immunogenicity of FMDV in target species (74), but are still unavailable to SVA infection. We found that neither Balb/c nor Kunming mice showed obvious signs after intramuscular $\left(7 \times 10^{6}\right.$ $\mathrm{TCID}_{50}$ /mouse $)$, subcutaneous $\left(2 \times 10^{7} \mathrm{TCID}_{50} / \mathrm{mouse}\right)$ or intraperitoneal $\left(2 \times 10^{7} \mathrm{TCID}_{50} /\right.$ mouse $)$ injection with the rescued SVA CH-LX-01-2016. Thus, overcoming these two challenges would greatly facilitate the development of SVA vaccines.

\section{CONCLUSIONS}

It has been 5 years since SVA emergence in China in 2015, and nowadays more than half of the P.A.M.s have been reported to be affected by SVA infection. A few provinces, like Guangdong and Henan, have been repeatedly infected, and meanwhile SVAs in these provinces have undergone inter-genetic recombination events, consequently forming novel strains. To date, SVAs have evolved into five genetic clades in China at a fast evolutionary speed. As a result, virus progenies have conferred subclinical infection of animals in some areas (15). Unfortunately, there is still no commercially available vaccine. All of these will be great challenges for the prevention and control of SVA in China. In June 2018, the Ministry of Agriculture and Rural Affairs of China issued an official notice regarding strategies for prevention and control of SVA nationwide (75). This notice would shed light on implementation of effective countermeasures against SVA in China.

\section{AUTHOR CONTRIBUTIONS}

FL and HS: conceptualization. FL, QW, and YH: writingoriginal draft preparation. NW: formal analysis. HS: project administration. All authors contributed to the article and approved the submitted version.

\section{FUNDING}

This work was supported by the National Key R\&D Program for the 13th Five-Year Plan, China (2016YFD050110404) and the Shandong Key Research and Development Program, China (2019GNC106074).

\section{ACKNOWLEDGMENTS}

We gratefully thank Dr. Shangjin Cui at Institute of Animal Sciences, Chinese Academy of Agricultural Sciences, for offering clinical figures to us. We also thank Dr. Bo Ni and Dr. Zhi Zhang at China Animal Health and Epidemiology Center for their opinions shared with us. 


\section{REFERENCES}

1. Zhang X, Zhu Z, Yang F, Cao W, Tian H, Zhang K, et al. Review of seneca valley virus: a call for increased surveillance and research. Front Microbiol. (2018) 9:940. doi: 10.3389/fmicb.2018.00940

2. Reddy PS, Burroughs KD, Hales LM, Ganesh S, Jones BH, Idamakanti N, et al. Seneca valley virus, a systemically deliverable oncolytic picornavirus, and the treatment of neuroendocrine cancers. J Natl Cancer Inst. (2007) 99:1623-33. doi: 10.1093/jnci/djm198

3. Wadhwa L, Hurwitz MY, Chevez-Barrios P, Hurwitz RL. Treatment of invasive retinoblastoma in a murine model using an oncolytic picornavirus. Cancer Res. (2007) 67:10653-6. doi: 10.1158/0008-5472.CAN-07-2352

4. Pasma T, Davidson S, Shaw SL. Idiopathic vesicular disease in swine in manitoba. Can Vet J. (2008) 49:84-5.

5. Singh K, Corner S, Clark S, Scherba G, Fredrickson R. Seneca valley virus and vesicular lesions in a pig with idiopathic vesicular disease. J Vet Sci Technol. (2012) 3:1-3. doi: 10.4172/2157-7579.1000123

6. Vannucci FA, Linhares DC, Barcellos DE, Lam HC, Collins J, Marthaler D. Identification and complete genome of seneca valley virus in vesicular fluid and sera of pigs affected with idiopathic vesicular disease, Brazil. Transbound Emerg Dis. (2015) 62:589-93. doi: 10.1111/tbed.12410

7. Leme RA, Oliveira TE, Alcantara BK, Headley SA, Alfieri AF, Yang M, et al. Clinical manifestations of senecavirus a infection in neonatal pigs, Brazil, 2015. Emerg Infect Dis. (2016) 22:1238-41. doi: 10.3201/eid2207.151583

8. Leme RA, Oliveira TES, Alfieri AF, Headley SA, Alfieri AA. Pathological, immunohistochemical and molecular findings associated with senecavirus a-induced lesions in neonatal piglets. J Comp Pathol. (2016) 155:14555. doi: 10.1016/j.jcpa.2016.06.011

9. Leme RA, Zotti E, Alcantara BK, Oliveira MV, Freitas LA, Alfieri AF, et al. Senecavirus a: an emerging vesicular infection in brazilian pig herds. Transbound Emerg Dis. (2015) 62:603-11. doi: 10.1111/tbed.12430

10. Wu Q, Zhao X, Bai Y, Sun B, Xie Q, Ma J. The first identification and complete genome of senecavirus a affecting pig with idiopathic vesicular disease in China. Transbound Emerg Dis. (2017) 64:1633-40. doi: 10.1111/tbed.12557

11. Wang M, Chen L, Pan S, Mou C, Shi K, Chen Z. Molecular evolution and characterization of novel seneca valley virus (SVV) strains in South China. Infect Genet Evol. (2019) 69:1-7. doi: 10.1016/j.meegid.2019.01.004

12. Liu J, Zha Y, Li H, Sun Y, Wang F, Lu R, et al. Novel recombinant seneca valley virus isolated from slaughtered pigs in guangdong province. Virol Sin. (2019) 34:722-4. doi: 10.1007/s12250-019-00139-8

13. Guo Z, Chen XX, Ruan H, Qiao S, Deng R, Zhang G. Isolation of three novel senecavirus a strains and recombination analysis among senecaviruses in China. Front Vet Sci. (2020) 7:2. doi: 10.3389/fvets.2020.00002

14. Wang Z, Zhang X, Yan R, Yang P, Wu Y, Yang D, et al. Emergence of a novel recombinant seneca valley virus in central China, 2018. Emerg Microbes Infect. (2018) 7:180. doi: 10.1038/s41426-018-0183-1

15. Zhang Z, Zhang L, Zhang M, Liu S, Zhang F, Dong Y, et al. Isolation of senecavirus a from clinical healthy pigs (in Chinese). Chin J Prev Vet Med. (2019) 41:418-21. doi: 10.3969/j.issn.1008-0589.201809028

16. Zhang Z, Ni B, Zhang L, Zhang H, Zhang F, Liu S, et al. Complete genome sequence of a novel senecavirus a isolate from an asymptomatic pig in China. Microbiol Resour Announc. (2019) 8:e01660-18. doi: 10.1128/MRA.01660-18

17. Strauss M, Jayawardena N, Sun E, Easingwood RA, Burga LN, Bostina M. Cryo-electron microscopy structure of seneca valley virus procapsid. J Virol. (2018) 92:e01927-17. doi: 10.1128/JVI.01927-17

18. Hellen CU, de Breyne S. A distinct group of hepacivirus/pestivirus-like internal ribosomal entry sites in members of diverse picornavirus genera: evidence for modular exchange of functional noncoding RNA elements by recombination. J Virol. (2007) 81:5850-63. doi: 10.1128/JVI.02403-06

19. Willcocks MM, Locker N, Gomwalk Z, Royall E, Bakhshesh M, Belsham GJ, et al. Structural features of the Seneca Valley virus internal ribosome entry site (IRES) element: a picornavirus with a pestivirus-like IRES. J Virol. (2011) 85:4452-61. doi: 10.1128/JVI.01107-10

20. Wu Q, Zhao X, Chen Y, He X, Zhang G, Ma J. Complete genome sequence of seneca valley virus CH-01-2015 identified in China. Genome Announc. (2016) 4:e01509-15. doi: 10.1128/genomeA.01509-15

21. Sun D, Chen S, Cheng A, Wang M. Roles of the picornaviral 3C proteinase in the viral life cycle and host cells. Viruses. (2016) 8:82. doi: 10.3390/v8030082
22. Hales LM, Knowles NJ, Reddy PS, Xu L, Hay C, Hallenbeck PL. Complete genome sequence analysis of seneca valley virus-001, a novel oncolytic picornavirus. J Gen Virol. (2008) 89(Pt 5):1265-75. doi: 10.1099/vir.0. 83570-0

23. Liu C, Li X, Liang L, Li J, Cui S. Isolation and phylogenetic analysis of an emerging senecavirus A in China, 2017. Infect Genet Evol. (2019) 68:7783. doi: 10.1016/j.meegid.2018.12.009

24. Wang S, Wang D, Zhao Y, Xie C, Zhao X, Ma Z, et al. Establishment and application of fluorescence quantitative RT-PCR assay for detection of senecavirus A (in Chinese). Chin J Prev Vet Med. (2019) 41:479-83. doi: 10.3969/j.issn.1008-0589.201809031

25. Houston E, Temeeyasen G, Piñeyro PE. Comprehensive review on immunopathogenesis, diagnostic and epidemiology of senecavirus A. Virus Res. (2020) 286:198038. doi: 10.1016/j.virusres.2020.1 98038

26. Joshi LR, Fernandes MH, Clement T, Lawson S, Pillatzki A, Resende TP, et al. Pathogenesis of senecavirus A infection in finishing pigs. J Gen Virol. (2016) 97:3267-79. doi: 10.1099/jgv.0.000631

27. Zhao X, Wu Q, Bai Y, Chen G, Zhou L, Wu Z, et al. Phylogenetic and genome analysis of seven senecavirus A isolates in China. Transbound Emerg Dis. (2017) 64:2075-82. doi: 10.1111/tbed.12619

28. Qian S, Fan W, Qian P, Chen H, Li X. Isolation and full-genome sequencing of seneca valley virus in piglets from China, 2016. Virol J. (2016) 13:173. doi: 10.1186/s12985-016-0631-2

29. Wang H, Li C, Zhao B, Yuan T, Yang D, Zhou G, et al. Complete genome sequence and phylogenetic analysis of senecavirus A isolated in Northeast China in 2016. Arch Virol. (2017) 162:3173-6. doi: 10.1007/s00705-017-3480-4

30. Zhu Z, Yang F, Chen P, Liu H, Cao W, Zhang K, et al. Emergence of novel seneca valley virus strains in China, 2017. Transbound Emerg Dis. (2017) 64:1024-9. doi: 10.1111/tbed.12662

31. Wang HB, Tian B, Lv HL, Wang F, Zhang T, Wang CY, et al. Emergence and complete genome of senecavirus A in pigs of henan province in China, 2017. Pol J Vet Sci. (2019) 22:187-90. doi: 10.24425/pjvs.2018.125612

32. Li X. Isolation of CH-LZ-2017 Strain and Establishment of qRT-PCR Detection methods for SVV (in Chinese). Lanzhou: Gansu Agricultural University. (2018).

33. Wang $H$, Niu C, Nong Z, Quan D, Chen Y, Kang O, et al. Emergence and phylogenetic analysis of a novel seneca valley virus strain in the guangxi province of China. Res Vet Sci. (2020) 130:207-11. doi: 10.1016/j.rvsc.2020.03.020

34. Niu C, Wang H, Nong Z, Quan D, Zeng Y, Ren T, et al. Isolation and Identification of Seneca Valley Virus in Guangxi Province (in Chinese). Chinese J Anim Infect Dis. (in press).

35. Sun Y, Cheng J, Wu RT, Wu ZX, Chen JW, Luo Y, et al. Phylogenetic and genome analysis of 17 novel senecavirus a isolates in guangdong province, 2017. Front Vet Sci. (2018) 5:314. doi: 10.3389/fvets.2018.00314

36. Liu J, Ren X, Li Z, Xu G, Lu R, Zhang K, et al. Genetic and phylogenetic analysis of reemerged novel seneca valley virus strains in guangdong province, 2017. Transbound Emerg Dis. (2018) 65:614-7. doi: 10.1111/tbed.12839

37. Chen P, Yang F, Cao W, Liu H, Zhang K, Liu X, et al. The distribution of different clades of seneca valley viruses in guangdong province, China. Virol Sin. (2018) 33:394-401. doi: 10.1007/s12250-0180056-8

38. Zhang Z, Zhang L, Zhang F, Liu S, Dong Y, Zhang H, et al. Retrospective surveillance for senecavirus a in porcine samples in China from 2016 to 2018 (in Chinese). China Anim Health Inspect. (2019) 36:1-6.

39. Mu G, Lu S, Zhang Z, Lin H. Epidemic surveillance of SVA for risk analysis in jilin province (in Chinese). Jilin Anim Husband Vet Med. (2019) 40:73-4.

40. Dai L, Wang J, Fang L, Zhang Y, Li G, Xie G, et al. A seroepidemiological investigation on senecavirus A in swine in hainan province in 2018 (in Chinese). China Anim Health Inspect. (2019) 36:8-11.

41. Bai J, Fan H, Zhou E, Li L, Li S, Yan J, et al. Pathogenesis of a senecavirus a isolate from swine in shandong province, China. Vet Microbiol. (2020) 242:108606. doi: 10.1016/j.vetmic.2020.108606

42. Borderia AV, Rozen-Gagnon K, Vignuzzi M. Fidelity variants and RNA quasispecies. Curr Top Microbiol Immunol. (2016) 392:303-22. doi: 10.1007/82_2015_483 
43. Lukashev AN. Recombination among picornaviruses. Rev Med Virol. (2010) 20:327-37. doi: 10.1002/rmv.660

44. Liu F, Huang Y, Wang Q, Shan H. Construction of eGFP-tagged senecavirus A for facilitating virus neutralization test and antiviral assay. Viruses. (2020) 12:E283. doi: 10.3390/v12030283

45. Chen L, Zhang J, Wang M, Pan S, Mou C, Chen Z. Pathogenicity of two Chinese seneca valley virus (SVV) strains in pigs. Microb Pathog. (2019) 136:103695. doi: 10.1016/j.micpath.2019.103695

46. Zhang H, Chen P, Hao G, Liu W, Chen H, Qian P, et al. Comparison of the pathogenicity of two different branches of senecavirus a strain in China. Pathogens. (2020) 9:39. doi: 10.3390/pathogens9010039

47. Lai L, Liu X, Tan L, Ye S, Qiu L, Chen S, et al. Genome sequencing and pathogenicity analysis of senecavirus A, SVA-FJLY (in Chinese). Acta Vet Zootech Sin. (2020) 51:820-6. doi: 10.11843/j.issn.0366-6964.2020.04.018

48. Yang M, van Bruggen $\mathrm{R}, \mathrm{Xu} \mathrm{W}$. Generation and diagnostic application of monoclonal antibodies against seneca valley virus. J Vet Diagn Invest. (2012) 24:42-50. doi: 10.1177/1040638711426323

49. Goolia M, Vannucci F, Yang M, Patnayak D, Babiuk S, Nfon CK. Validation of a competitive ELISA and a virus neutralization test for the detection and confirmation of antibodies to Senecavirus A in swine sera. J Vet Diagn Invest. (2017) 29:250-3. doi: 10.1177/1040638716683214

50. Zhang J, Nfon C, Tsai CF, Lee CH, Fredericks L, Chen Q, et al. Development and evaluation of a real-time RT-PCR and a field-deployable RT-insulated isothermal PCR for the detection of seneca valley virus. BMC Vet Res. (2019) 15:168. doi: 10.1186/s12917-019-1927-4

51. Wang Y, Das A, Zheng W, Porter E, Xu L, Noll L, et al. Development and evaluation of multiplex real-time RT-PCR assays for the detection and differentiation of foot-and-mouth disease virus and seneca valley virus 1 . Transbound Emerg Dis. (2019) 67:604-16. doi: 10.1111/tbed.13373

52. Fowler VL, Ransburgh RH, Poulsen EG, Wadsworth J, King DP, Mioulet V, et al. Development of a novel real-time RT-PCR assay to detect seneca valley virus-1 associated with emerging cases of vesicular disease in pigs. J Virol Methods. (2017) 239:34-7. doi: 10.1016/j.jviromet.2016.10.012

53. Feronato C, Leme RA, Diniz JA, Agnol AMD, Alfieri AF, Alfieri AA. Development and evaluation of a nested-PCR assay for senecavirus A diagnosis. Trop Anim Health Prod. (2018) 50:337-44. doi: 10.1007/s11250-017-1436-Z

54. Pinheiro-de-Oliveira TF, Fonseca-Junior AA, Camargos MF, LaguardiaNascimento M, Giannattasio-Ferraz S, Cottorello ACP, et al. Reverse transcriptase droplet digital PCR to identify the emerging vesicular virus senecavirus A in biological samples. Transbound Emerg Dis. (2019) 66:13609. doi: $10.1111 /$ tbed. 13168

55. Bracht AJ, O'Hearn ES, Fabian AW, Barrette RW, Sayed A. Realtime reverse transcription PCR assay for detection of senecavirus $\mathrm{A}$ in swine vesicular diagnostic specimens. PLoS ONE. (2016) 11:e0146211. doi: 10.1371/journal.pone.0146211

56. Dall Agnol AM, Otonel RAA, Leme RA, Alfieri AA, Alfieri AF. A taqmanbased qRT-PCR assay for senecavirus A detection in tissue samples of neonatal piglets. Mol Cell Probes. (2017) 33:28-31. doi: 10.1016/j.mcp.2017.03.002

57. Fan H, Li L, Jiang P, Wang X, Li Y, Bai J. Establishment and application of a RT-PCR assay for detection of senecavirus A (SVA) (in Chinese). Acta Vet Zootech Sin. (2019) 50:1312-8. doi: 10.11843/j.issn.0366-6964.2019. 06.022

58. Liu J, Zha Y, Lu R, Ren X, Li Z, Li H, et al. Establishment and primary application of real-time RT-PCR for the detection of porcine senecavirus A (in Chinese). Chin Vet Sci. (2019) 49:274-9. doi: 10.16656/j.issn.1673-4696.2019.0041

59. Zhang Z, Zhang $\mathrm{Y}$, Lin $\mathrm{X}$, Chen Z, Wu S. Development of a novel reverse transcription droplet digital PCR assay for the sensitive detection of senecavirus A. Transbound Emerg Dis. (2019) 66:517-25. doi: 10.1111/tbed.13056

60. Zeng F, Cong F, Liu X, Lian Y, Wu M, Xiao L, et al. Development of a real time loop-mediated isothermal amplification method for detection of senecavirus A. J Virol Methods. (2018) 261:98-103. doi: 10.1016/j.jviromet.2018. 08.005

61. Li J, Liang W, Xu S, Shi J, Zhou X, Liu B, et al. Rapid and sensitive detection of senecavirus A by reverse transcription loop-mediated isothermal amplification combined with a lateral flow dipstick method. PLoS ONE. (2019) 14:e0216245. doi: 10.1371/journal.pone.0216245

62. Fan X, Song Z, Zhao Y, Zhao M, Dong Y, Zhang Y, et al. Establishment of recombinase polymerase amplification-lateral flow dipstick for the detection of seneca valley virus (in Chinese). Chin J Prev Vet Med. (2018) 40:406-10. doi: 10.3969/j.issn.1008-0589.201708027

63. Fan X, Hadeng C, Wang Y, Wang J, Liu C, Chi T, et al. Establishment of a real-time fluorescent recombinase polymerase amplification (RPA) for the detection of seneca valley virus (in Chinese). China Anim Health Inspect. (2017) 34:81-6.

64. Zhao Y, Yan R, Wang S, Ma Z, Ban F, Zhao X, et al. Establishment and preliminary application of indirect ELISA based on structural protein VP1 of senecavirus A (in Chinese). Chin J Prev Vet Med. (2019) 41:818-23. doi: 10.3969/j.issn.1008-0589.201901049

65. Shen S, Xu L, Li R, Shi X, Wang H, Miao T, et al. Establishment of blocking ELISA detection method of seneca virus type A VP2 protein (in Chinese). Heilongjiang Anim Sci Vet Med. (2020) 14:87-90. doi: 10.13881/j.cnki.hljxmsy.2019.09.0090

66. Shen S, Shi X, Li R, Ma Y, Qin J, Wang E. Development of an indirect ELISA for detection of VP2 protein against seneca virus A (in Chinese). Chin Vet Sci. (2020) 50:152-8. doi: 10.16656/j.issn.1673-4696.2020.0013

67. Sharma B, Fernandes MHV, de Lima M, Joshi LR, Lawson S, Diel DG. A novel live attenuated vaccine candidate protects against heterologous senecavirus A challenge. Front Immunol. (2019) 10:2660. doi: 10.3389/fimmu.2019. 02660

68. Yang F, Zhu Z, Cao W, Liu H, Zhang K, Tian H, et al. Immunogenicity and protective efficacy of an inactivated cell culture-derived seneca valley virus vaccine in pigs. Vaccine. (2018) 36:841-6. doi: 10.1016/j.vaccine.2017.12.055

69. Wei T, Yang F, Zhang W, Wang Z, Sun X, Zheng H. Construction of eukaryotic expression plasmid for P12A-3C gene of senecavirus $\mathrm{A}$ and immunity experiments in mouse (in Chinese). Chin Vet Sci. (2020) 50:861-8. doi: 10.16656/j.issn.1673-4696.2020.0101

70. Lu Y, Dong H, Li J, Li L, Wang M, Liu H, et al. Enhanced protective immune response of foot-and-mouth disease vaccine through DNA-loaded virus-like particles. Microb Pathog. (2020) 143:104130. doi: 10.1016/j.micpath.2020.104130

71. Liu X, Fang Y, Zhou P, Lu Y, Zhang Q, Xiao S, et al. Chimeric viruslike particles elicit protective immunity against serotype $\mathrm{O}$ foot-and-mouth disease virus in guinea pigs. Appl Microbiol Biotechnol. (2017) 101:490514. doi: 10.1007/s00253-017-8246-0

72. Xiao Y, Chen HY, Wang Y, Yin B, Lv C, Mo X, et al. Large-scale production of foot-and-mouth disease virus (serotype Asia1) VLP vaccine in Escherichia coli and protection potency evaluation in cattle. BMC Biotechnol. (2016) 16:56. doi: 10.1186/s12896-016-0285-6

73. Mo Y, Song P, Mu S, Dong H, Cao S, Guo H, et al. Prokaryotic expression of capsid protein of seneca virus type A and assembly of its pentamer (in Chinese). Chin Vet Sci. (2019) 49:969-76. doi: 10.16656/j.issn.1673-4696.2019.0098

74. Habiela M, Seago J, Perez-Martin E, Waters R, Windsor M, Salguero FJ, et al. Laboratory animal models to study foot-and-mouth disease: a review with emphasis on natural and vaccine-induced immunity. J Gen Virol. (2014) 95(Pt 11):2329-45. doi: 10.1099/vir.0.068270-0

75. China: Ministry of Agriculture and Rural Affairs. (2018) Available online at: http://www.moa.gov.cn/gk/tzgg_1/tfw/201806/t20180620_6152740.html. (accessed June 20, 2018).

Conflict of Interest: The authors declare that the research was conducted in the absence of any commercial or financial relationships that could be construed as a potential conflict of interest.

Copyright (c) 2020 Liu, Wang, Huang, Wang and Shan. This is an open-access article distributed under the terms of the Creative Commons Attribution License (CC BY). The use, distribution or reproduction in other forums is permitted, provided the original author(s) and the copyright owner(s) are credited and that the original publication in this journal is cited, in accordance with accepted academic practice. No use, distribution or reproduction is permitted which does not comply with these terms. 